\title{
KERNFORSCHUNGSZENTRUM
}

\author{
KARLSRUHE
}

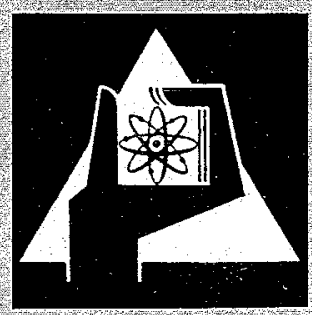





\title{
LETTERS TO THE EDITOR
}

\section{A CIRCUIT FOR PULSE SHAPE DISCRIMINATION}

\author{
W. SCHWEIMER
}

Zyklotron-Laboratorium, Kernforschungszentrum Karlsruhe

Received 3 November 1965

The scintillation materials $\mathrm{NaI}(\mathrm{Tl}), \mathrm{KI}(\mathrm{Tl}), \mathrm{CsI}(\mathrm{Tl})$, NE 150, NE 213, etc. show a dependence of the shape of the light pulses on the ionization density produced by the ionizing particles. Alexander and Goulding ${ }^{1}$ ) have reported one of the electronic principles to distinguish between different pulse shapes. It consists of an integration of the current pulses of a multiplier with a time constant between the two main decay time constants of the light pulses. After the integration, a clipping or slow differentiation causes a zero-crossing of the signal with a zero-crossing time depending on the amplitude relation between the slow and fast components of the light pulses.

Lately this principle has been successfully used for identification of electrons, protons, deuterons and alpha-particles in $\mathrm{NaI}(\mathrm{Tl}), \mathrm{KI}(\mathrm{Tl})$ and $\mathrm{CsI}(\mathrm{Tl})$ crystals ${ }^{2,3}$ ).

We have used a simple circuit for a NE 213 scintillator based on the Alexander and Goulding principle. The circuit has proved to be reliable and faultfree in the last two years. Fig. 1 shows the circuit diagram. The input signals are current pulses from the $11^{\text {th }}$ dynode of a XP 1040-multiplier. There are two output

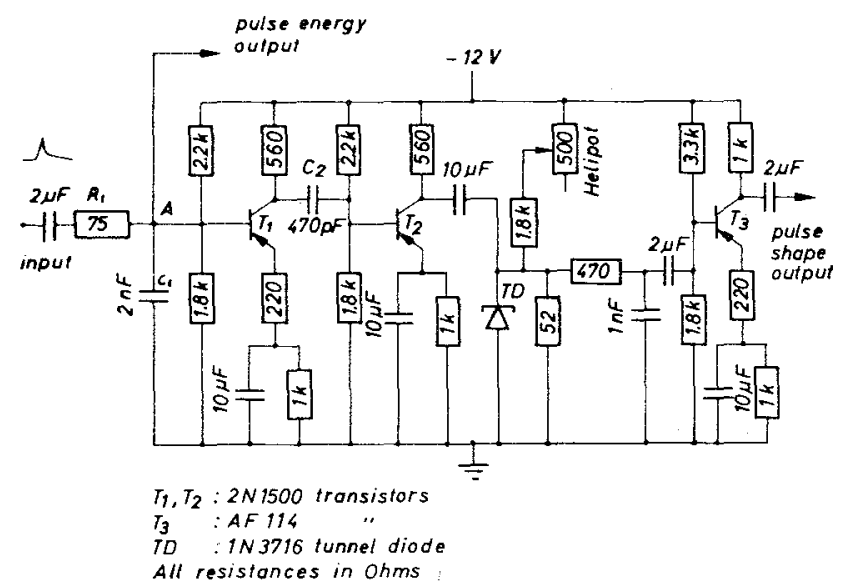

Fig. 1. Circuit diagram of the pulse shape discriminator. The input signals are current pulses of a dynode of a XP 1040 multiplier seeing a NE 213 scintillator. Output pulses are pulse shape analog pulses and energy analog pulses. signals, the pulse aisplitude of the first being shape dependent and the second one carrying the integrated charge of the input signal.

The input signal is integrated with the $\mathrm{R}_{1} \mathrm{C}_{1}$ integrator and then voltage amplified with transistor $T_{1}$. The capacitance $C_{2}$ causes the slow differentiation for the zero crossing. Transistor $T_{2}$ is a current amplifier and inverter. The tunnel diode network drives a positive current into the integrating $1 \mathrm{nF}$ capacitance during the time interval between the start and zerocrossing of the current pulse from $\mathrm{T}_{2}$.

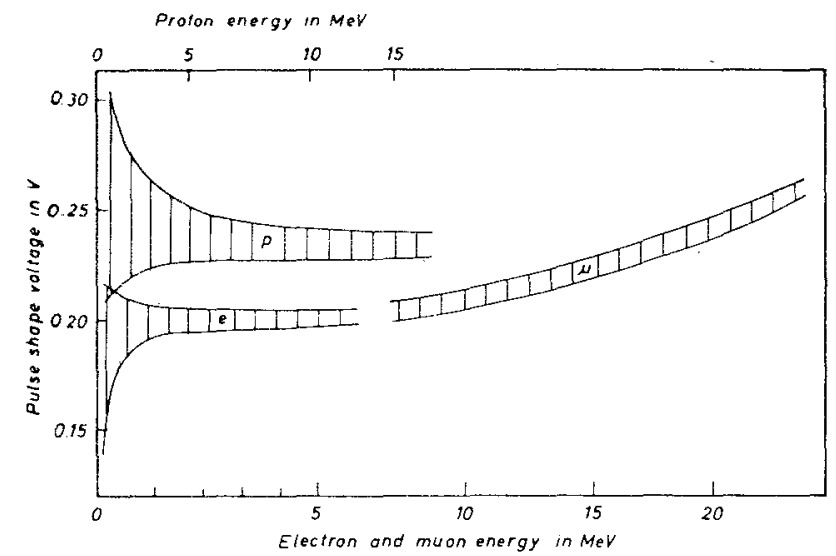

Fig. 2. Regions of the energy-pulse shape plane where electron, proton and cosmic muon events fall in.

The tunnel diode is normally in the valley point of its characteristic curve. A positive current from $T_{2}$ switches the tunnel diode to the Zener-region and when the current from $T_{2}$ approaches zero the voltage-current point on the characteristic curve of the tunnel diode approaches the peak point. If the $52 \Omega$ resistor and the helipot are correctly adjusted, the positive maximum of the integrated tunnel diode pulse is proportional to the zero-crossing time. Transistor $\mathrm{T}_{3}$ is a voltage amplifier.

The integrated charge of the input signal can be decoupled at point $A$ by an amplifier similar to $T_{3}$.

The $150 \mathrm{~ns}$ input integration-time-constant is large enough to make the stray capacitances negligible on 
the signal way between the multiplier dynode and the circuit input. The circuit can be soldered on one $105 \times 120 \mathrm{~mm}^{2}$ transistor card with all input and output connections through a 24 pole connector.

Fig. 2 shows the regions of the energy-pulse shape plane where electron, cosmic muon and proton events

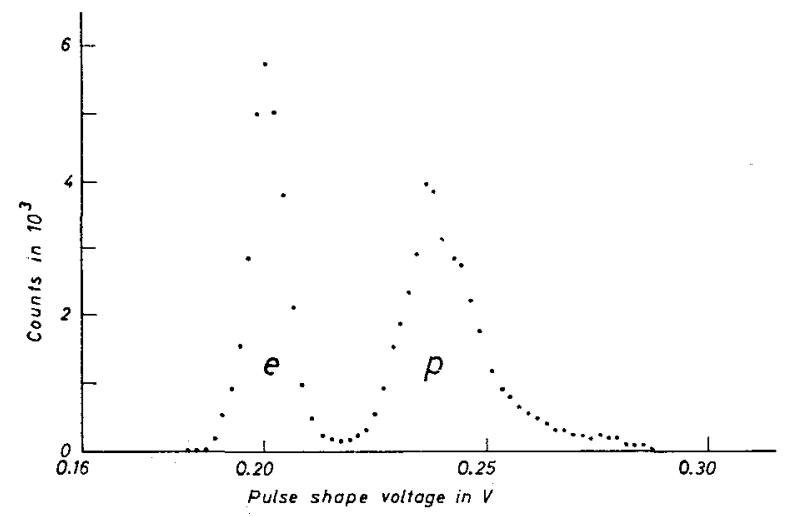

Fig. 3. Pulse shape analog amplitude distribution for a Po-Be source. Energy threshold was set at $0.47 \mathrm{MeV}$ electron or equivalent proton recoil energy. fall in. The electron and proton events were obtained from a Po-Be source as well as from the neutron beam of the Karlsruhe cyclotron. The muon events were obtained in a $120 \mathrm{~mm}$ diameter $120 \mathrm{~mm}$ thick NE 213 scintillator. The variation of the pulse shape signal in the upper energy region is a saturation effect of the fasi component of the light pulses in the multiplier or in the first stage of the circuit. This saturation makes the electron and muon events more "proton like".

Fig. 3 is shown for comparison with results of other circuits. It is the pulse shape signal amplitude distribution obtained with a NE 213 scintillator and a Po-Be source. A coincidence pulse from the energy signal was fed to the multichannel analyser for electron energies above $0.47 \mathrm{MeV}$ or equivalent proton recoil energies.

\section{References}

1) T. K. Alexander and F. S. Goulding, Nucl. Instr. and Meth. 13 (1961) 244

2) R. Bass, W. Kessel and G. Majoni, Nucl. Instr. and Meth. 30 (1964) 237; Nucl. Instr. and Meth. 34 (1965) 169.

3) R. Fülle, Gy. Máthé and D. Netzband, Nucl. Instr. and Meth. 35 (1965) 250. 